\title{
Endovascular extraction of a disconnected port-A catheter from inferior vena cava in a patient with chronic bilateral occlusion of femoroiliac veins: A case report
}

\author{
(D) Alireza Rai', (D) Mohammadreza Sobhiyeh² \\ ${ }^{1}$ Kermanshah University of Medical Sciences, Imam Ali Hospital, Clinical Research Development Center, Clinic of Cardiology, \\ Kermanshah, Iran \\ 2Kermanshah University of Medical Sciences, Imam Reza Hospital, Clinical Research Development Center, Clinic of Vascular \\ and Endovascular Surgery, Kermanshah, Iran
}

\section{Date submitted:}

26.12.2019

Date accepted:

27.03.2020

Online publication date:

15.09.2020

\section{Corresponding Author:}

Mohammadreza Sobhiyeh, MD, Kermanshah University of Medical Sciences, Imam Reza Hospital, Clinical Research Development Center, Clinic of Vascular and Endovascular Surgery, Kermanshah, Iran mreza.sobhiyeh@yahoo.com

ORCID:

orcid.org/0000-0002-7333-1316

Keywords: Venous port system, chemotherapy, migration, internal jugular vein

\begin{abstract}
Catheter detachment from a port may be seen due to various reasons, including operator errors, subsequent catheter manipulation during endovascular procedures, and trauma. For catheter removal, various tools including loop snare or basket catheter may be used with access via femoral or jugular veins. In this paper, we report the removal of a detached port-A catheter using a snare catheter from a jugular venous access in a patient with ilio-femoral venous occlusion. The main reason for reporting this case is the central venous catheter migration immediately after insertion and total occlusion of bilateral ilio-femoral veins due to unsuccessful manipulations previously.
\end{abstract}

\section{Introduction}

Percutaneously implanted venous port catheters provide permanent access for intravenous therapy and can be used for several years if handled correctly (1). Dislodgement of the catheter may be seen due to manipulation or trauma in less than $3 \%$ of patients (2). In cases of central venous catheter migration to the heart or inferior vena cava (IVC), endovascular retrieval using a snare is usually attempted through a jugular or femoral or subclavian access $(3,4)$. We report a case of a dislocated and migrated port-A catheter into the IVC in a young woman immediately after its implantation.

\section{Presentation of Case}

A 36-year-old woman with a previous history of rectal cancer received a port-A catheter in her right jugular vein for chemotherapy in 2017 at another hospital. Directly after implantation, the catheter was non-functional. Subsequent chest X-ray revealed a dislocated catheter migrated into the

${ }^{\odot}$ Copyright 2020 by the University of Health Sciences Turkey, Gülhane Faculty of Medicine / Gülhane Medical Journal published by Galenos Publishing House. 
right atrium (Figure 1). No further information was available about the details of the primary operation and the reason for unsuccessful retrieval of the catheter by interventional technique through bilateral femoral veins access. The patient had undergone unsuccessful attempts for extracting the catheter in an urban tertiary health care center twice. She was under oral anticoagulation with warfarin thereafter. The cause of anticoagulant therapy was bilateral development of iliofemoral vein thrombosis after two times of unsuccessful manipulations. The patient was referred to our hospital in 2018 for the extraction

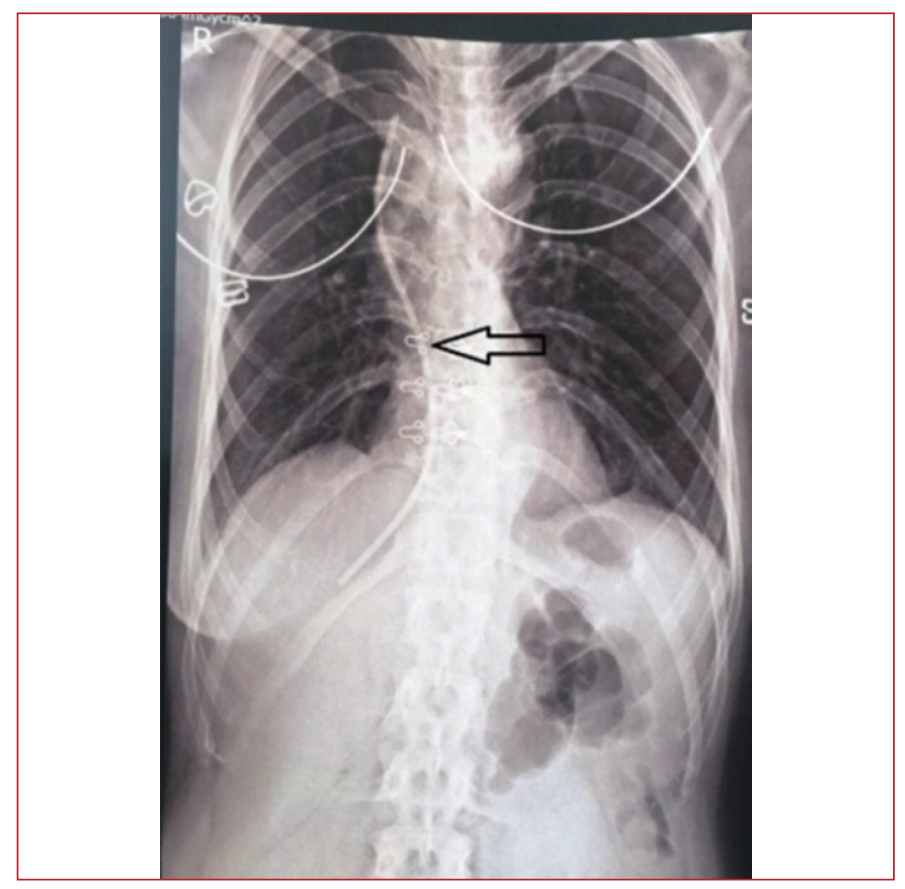

Figure 1. The port-A-catheter was dislodged in inferior vena cava and right atrium

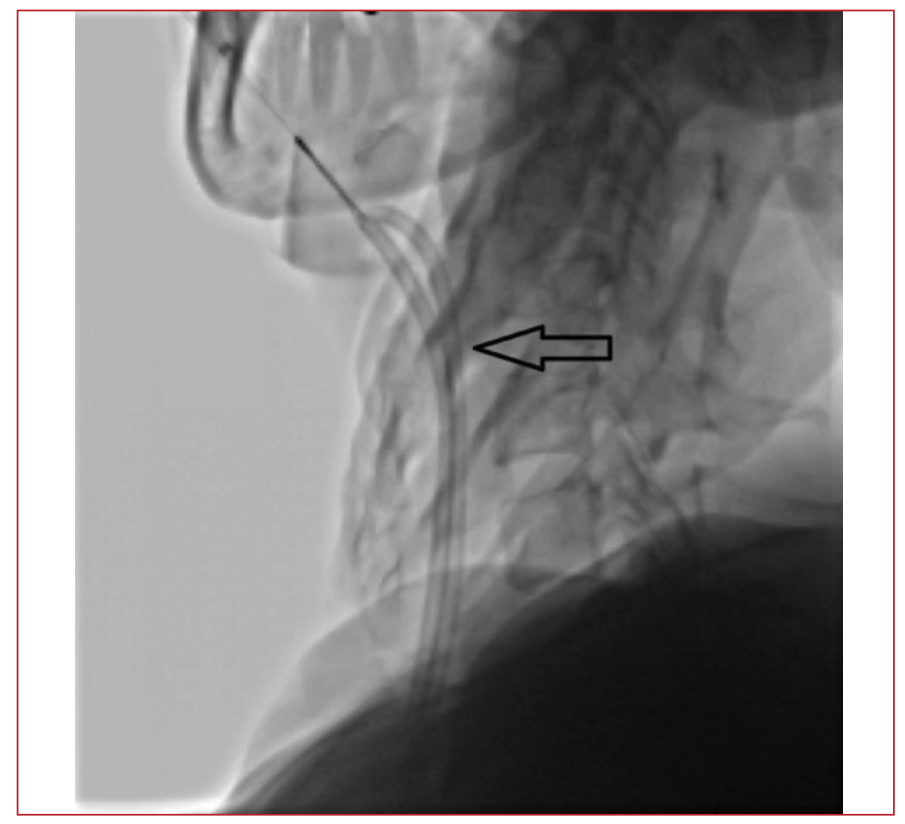

Figure 2. The port-A-catheter was captured and removed successfully of the foreign body and discontinued the oral anticoagulation due to newly developed gastrointestinal bleeding. The preoperative clinical and sonography assessment showed a bilateral occlusion of ilio-femoral veins. This finding was also noted on the previous venography as well as a partial recanalized occlusion of IVC below the renal veins. Because of the risk of bleeding and contraindication for oral anticoagulation, we did not intend to puncture the femoral veins and recanalization of the iliocaval tract as the first option. Instead, we planned to capture the catheter by snaring through right internal jugular vein (IJV). After cannulating the right IJV under ultrasound guidance, the catheter was removed with a large loop snare. During the withdrawal the catheter was folded and stuck to the sheath's introducer tip (Figure 2). We stopped pulling back the catheter and tried to remove it together with the sheath. However, due to the bulk of folded catheter, it was impossible to extract the catheter through puncture hole. To avoid damaging the IJV, a 1.5 centimeter incision under local anesthesia was made at the entry site of sheath (included cutaneous and subcutaneous fat) due to fibrosis and the catheter was removed successfully.

\section{Discussion}

Implantation of a port system is indicated if drugs are to be administered over a longer period of time (5). Although the catheter placement is relatively safe, several potential complications can occur including catheter dislodgement, catheter malposition, catheter compression and catheter fracture (2). The dislodged catheter scan migrates along the blood stream to the superior vena cava, the right atrium, the right ventricle, the main pulmonary artery or its branches $(3,4)$. The risk factors for intravascular thrombus formation in case of catheter migration include endothelial injury, hemodynamic flow changes within vessel and partial stasis $(5,6)$. Anticoagulation therapy alone may not prevent thrombus formation in the presence of a dislodged catheter. Before the procedure, color Doppler ultrasound of the femoral and jugular veins was initially done bilaterally, and also simple thoracic and abdominal radiography was obtained to determine definite catheter location.

Several percutaneous extraction techniques of dislocated or fragmented cardiovascular catheters like loop snare, a forceps, a guide wire and a basket catheter have been suggested (4). Use of loop snare is relatively safe and the most often reported technique (3). Our initial strategy was to snare and pull back the catheter through the right IJV access. In case of failure of the right and left jugular veins, we were prepared to use a femoral access and recanalize the iliac and infra renal IVC to increase the possibility of endovascular extraction of the catheter. We succeed in retrieval with triple-loop snare through the right IJV. The problem in this case was mainly one-year persistence of the catheter. Also, bilateral occlusion of the ilio-femoral 
vein excluded the chance of procedure exclusion. However, the dislocated catheter could not pass through the sheath or pulled out together with it, so a small incision (cutaneous and subcutaneous fat) was needed to avoid vessel injury.

In our reported case, due to two times of unsuccessful manipulation, thrombosis developed and the catheter was remained one year in the right atrium and IVC that led to fibrin sheath formation around the catheter and it was removed after one year. Our experience is that the dislocated catheter in the heart or IVC can easily be grasped using a loop snare even long after implantation. Nevertheless, the fibrin sheath around the catheter may hamper its extraction and can cause damage to vessel wall. Therefore, extraction of migrated catheter of IJV route is feasible, but the presence of thrombosis and fibrin sheath may reduce the chance of successful intervention.

\section{Conclusion}

Intravascular migration of central catheter is sometimes associated with severe and rare complications of indwelling intravenous catheters such as IVC thrombosis, that the anticoagulation administration alone cannot prevent catheter thrombosis. Therefore, after central catheter migration, immediately should be retrieved by experience interventional center.

\section{Acknowledgement}

In addition, the authors would like to thank the clinical Research Development center of Imam Reza Hospital, Kermanshah University of Medical sciences for consulting services.

\section{Ethics}

Informed Consent: Consent form was filled out by the patient.
Peer-review: Externally peer-reviewed.

\section{Authorship Contributions}

Concept: A.R., Design: A.R., Data Collection or Processing: A.R., Analysis or Interpretation: M.S., Literature Search: M.S., Writing: A.R., M.S.

Conflict of Interest: No conflict of interest was declared by the authors.

Financial Disclosure: The authors declared that this study received no financial support.

\section{References}

1. Akaraborworn $\mathrm{O}$. A review in emergency central venous catheterization. Chin J Traumatol. 2017;20:137-140.

2. Massmann A, Jagoda $P$, Kranzhoefer N, Buecker A. Percutaneous Re-positioning of Dislocated Port-Catheters in Patients with Dysfunctional Central-Vein Port-Systems. Ann Surg Oncol. 2015;22:4124-4129.

3. Peng J, Zhang XM, Yang L, et al. A Novel Two-Step Technique for Retrieving Fractured Peripherally Inserted Central Catheter Segments Migrating into the Heart or the Pulmonary Artery. Biomed Res Int. 2016;2016:7814529.

4. Teragawa H, Sueda T, Fujii Y, et al. Endovascular technique using a snare and suture for retrieving a migrated peripherally inserted central catheter in the left pulmonary artery. World J Cardiol. 2013;5:369-372.

5. Carr PJ, Rippey JC. Upper extremity deep vein thrombosis: a complication of an indwelling peripherally inserted central venous catheter. Clin Case Rep. 2015;3:170-174.

6. Chopra V, Anand S, Krein SL, Chenoweth C, Saint S. Bloodstream infection, venous thrombosis, and peripherally inserted central catheters: reappraising the evidence. Am J Med. 2012;125:733-741. 